\title{
FIBROMYALGIA: THE PATIENT'S PERCEPTION
}

\author{
Elisa Noskoski ${ }^{1, \star}$, Christine Maria Kaiser Leitner ${ }^{1}$, Maikelli Simes ${ }^{1}$, Kenia Rodrigues de Andrade ${ }^{1}$, Samuel Spiegelberg Zuge ${ }^{1}$ \\ 1.Universidade Comunitária da Região de Chapecó, Chapecó (SC), Brazil. \\ *Corresponding author: elisa.noskoski@unochapeco.edu.br
}

\section{BACKGROUND}

Events that cause tension, both physical, mental or emotional are triggered by stressors. These act by activating emotions such as fear, ambition, anger, guilt, which in turn have an ability to generate physical consequences. It is known that fibromyalgia is a syndrome whose pain and hypersensitivity are the main symptoms. This pathology does not have a cure and its treatment seeks to alleviate the symptoms, using a pharmacological and biopsychosocial approach.

\section{METHODS}

This is a cross-sectional, qualitative study. The research included those diagnosed with fibromyalgia over the age of 18 years. Data were collected through the application of an online questionnaire, formulated in Google Forms, with the two questions: "How was it to receive the diagnosis of fibromyalgia?" and "What is it like to live with fibromyalgia?" Without any delimitation, the patient was able to include his feelings and reality in his answer. The data analysis occurred by reading the answers, analyzing the frequency that the words appeared in the answers and formulating a word cloud, highlighting the most frequent ones.

\section{RESULTS}

Among 427 participants, there was a predominance of females: 423 (99.1\%), with only 4 (0.9\%) men. Regarding age, most were in the range of 30 to 44 years $(235 ; 55 \%)$ followed by the range of 45 to 54 years $(119,27.9 \%)$. When asked about the moment of diagnosis, the words "sad" and "relief" stand out, mentioned 48 and 41 times, respectively. Also, words like "difficult", "scary", "bad", "hopeless" were used frequently. It is noticed that despite the difficulty of diagnosis, many expressed comfort for having an explanation for that excessive pain. Regarding living with fibromyalgia, negative feelings predominate in the answers, such as "difficult", "horrible" and "terrible". The pathology was highlighted as a generator of disabilities, with a predominance of pain and fatigue, feelings of hopelessness, depression, uncertainty, exhaustion and sadness. The lack of understanding of family and society was highlighted as a complicating factor for fibromyalgia.

\section{CONCLUSION}

Fibromyalgia patients need care and attention. As for the diagnosis, sadness was a relevant feeling mentioned; however, being relieved demonstrates how the pain of these patients is misunderstood by others in different situations. Regarding living with the disease, the difficulties with the impact on daily life lead to other reflexes and feelings, such as discouragement and a feeling of incapacity.

\section{KEYWORDS}

Pain, Diagnosis, Living, Fatigue. 